\title{
REVIEW
}

\section{Bench-to-bedside review: An approach to hemodynamic monitoring - Guyton at the bedside}

Sheldon Magder*

\begin{abstract}
Hemodynamic monitoring is used to identify deviations from hemodynamic goals and to assess responses to therapy. To accomplish these goals one must understand how the circulation is regulated. In this review I begin with an historical review of the work of Arthur Guyton and his conceptual understanding of the circulation and then present an approach by which Guyton's concepts can be applied at the bedside. Guyton argued that cardiac output and central venous pressure are determined by the interaction of two functions: cardiac function, which is determined by cardiac performance; and a return function, which is determined by the return of blood to the heart. This means that changes in cardiac output are dependent upon changes of one of these two functions or of both. I start with an approach based on the approximation that blood pressure is determined by the product of cardiac output and systemic vascular resistance and that cardiac output is determined by cardiac function and venous return. A fall in blood pressure with no change in or a rise in cardiac output indicates that a decrease in vascular resistance is the dominant factor. If the fall in blood pressure is due to a fall in cardiac output then the role of a change in the return function and cardiac function can be separated by the patterns of changes in central venous pressure and cardiac output. Measurement of cardiac output is a central component to this approach but until recently it was not easy to obtain and was estimated from surrogates. However, there are now a number of non-invasive devices that can give measures of cardiac output and permit the use of physiological principles to more rapidly appreciate the primary pathophysiology behind hemodynamic abnormalities and to provide directed therapy.
\end{abstract}

*Correspondence: sheldon.magder@muhc.mcgill.ca McGill University Health Centre, Royal Victoria Hospital, 687 Pine Avenue West, Montreal, Quebec, Canada H3A 1A1

\section{Introduction}

A basic premise for the management of hemodynamic instability is that a response to a therapy can only be in the range of the physiologically possible. It is thus important to have a physiological construct of the circulation to interpret the information provided by monitoring devices and to provide rational responses. Arthur Guyton's analysis of the circulation provides such a construct. I will first review the concepts Guyton developed and then indicate how these concepts can be used to provide an approach to monitoring and management of hemodynamic abnormalities in critically ill patients.

\section{What did Arthur Guyton teach us?}

Arthur Guyton presented a paradigm shift from the prevailing view at the time of how the circulation works. Before Guyton, control of cardiac output was primarily considered in terms of heart rate, stroke volume, and function of the heart [1] and little thought was given to the importance of how blood gets back to the heart [2]. In an early paper, Guyton credited Ernest Starling for appreciating that output from the heart is dependent upon the return of venous blood and that venous return is dependent upon the pressure upstream to the heart in the systemic circulation, which Starling called mean systemic pressure. Guyton's comments in this paper are typical of his generosity of spirit, because Starling had not dealt with the mechanics of the systemic circulation or the factors that determine flow back to the heart; the concept of venous return and its determinants awaited Guyton. His key statement - which seems so obvious today, but is still challenged by some - was best enunciated in his 1957 paper that first reported his experiments in which he created venous return curves [3]:

'When a change occurs in the hemodynamics of the circulatory system one cannot predict what will happen to the cardiac output unless he takes into consideration both the effect of this change on the ability of the heart to pump blood and also the tendency for blood to return to the heart from the blood vessels'.

Based on this concept, cardiac output is determined by the function of the heart - or, as Guyton called it, a cardiac response curve - and by the function of the 
circuit, which determines the return of blood to the heart and which Guyton depicted as a venous return curve. Guyton first clarified the concept of mean circulatory filling pressure (MCFP) and established an experimental approach to measure it [4]. He was then able to construct venous return curves and analyze the factors that affect them [3]. Finally, to my mind his most important legacy, he provided a graphical tool that allows one to analyze the complex interactions that occur between the function of the heart (cardiac function) and the return of blood to the heart (venous return function) $[2,5,6]$. These curves provide a powerful conceptual tool for analyzing these interactions and understanding the broad physiological responses.

\section{Importance of compliance}

MCFP is the pressure in the vasculature when there is no flow and it is determined by the volume that distends all of the elastic structures in the circulation and the sum of all their compliances. Guyton measured MCFP by arresting the circulation and using a pump to rapidly equilibrate the arterial and venous pressures.

Vascular compliance is a static measurement; that is, it is a characteristic of the system even when there is no flowing blood. Yet this static property is a critical determinant of the amount of blood that can flow around the circulation. The reasoning is as follows. Flow occurs based on a pressure gradient from the beginning to the end of a circuit. In the cardiovascular system, pressure and flow are pulsatile because of the cyclic nature of cardiac contractions. If the walls of a closed loop system are noncompliant (stiff), an increase in pressure at the beginning of the circuit, such as occurs with a contracting heart, would be immediately transmitted throughout the system and there would be no gradient for a flow wave to occur. A flow wave can occur only if some part of the circuit transiently takes up volume and then releases it; that is, the circuit must have a compliant region that allows a change in volume for a change in pressure.

In the vasculature, all vessels have some compliance, but the small veins and venules, especially those in the splanchnic circulation, have a compliance that is 30 to 40 times greater than the compliance of other vessels. Accordingly, approximately $70 \%$ of stressed blood volume resides in the venules and veins at a pressure of around 8 to $10 \mathrm{mmHg}$. I must emphasize that I have referred to 'the volume that stretches the vascular walls', because not all of the total blood volume acts to distend the elastic walls of the circulation. Under basal conditions, only about $30 \%$ of total blood volume actually stretches the vessel walls and creates MCFP [7]. This volume is called the stressed volume, whereas the volume that rounds out the vessels but does not produce a pressure is called the unstressed volume. Contraction of smooth muscles in the walls of veins and venules by neuro-humeral mechanisms can recruit unstressed volume into stressed volume and increase MCFP $[8,9]$ by shifting the vascular volume-pressure curve to the left along the $x$ axis. Importantly, this critical hemodynamic reserve of unstressed volume cannot be measured by any monitoring device.

When there is blood flow in the circulation, blood volume redistributes among all of the elastic compartments of the circulation based on their compliances and the resistances separating them, but the pressure in the venous compliant region does not usually change very much because its compliance is so large relative to other compartments. However, this volume changes when left heart function is poor and right heart function is maintained for volume redistribution to the pulmonary circuit [10]. Even though MCFP is the determinant of the capacity of the system for flow, the primary determinant of venous return under flow conditions is the pressure in the systemic veins. To distinguish the pressure in the systemic venous reservoir under flow conditions from the static pressure of the vasculature when there is no flow and complete equilibration of the pressures in all compartments, the pressure in systemic veins is called mean systemic filling pressure (MSFP). This is usually very close to, but not always the same as, MCFP.

An infusion of volume increases MSFP and thereby increases the gradient for venous return. This increase can also occur by recruitment of unstressed volume into stressed volume by baroreceptor-induced activation of sympathetic output or by the infusion of $\alpha$-adrenergic agonists [9,11-13]. This process is called a change in capacitance, which refers to total pressure for total volume. The slope of the relationship of the vascular pressure-volume relationship is elastance, which is the inverse of compliance. In the physiological range this slope usually does not change. As already noted, the reserve of unstressed volume cannot be measured in an intact person and must be predicted from a patient's clinical status by considering factors that could have resulted in volume loss and use of the body's reserves in unstressed volume. Furthermore, arterial pressure does not determine MSFP because MSFP is determined by the volume that flows into the veins relative to the volume that leaves the veins. The upstream pressure needed for a given flow is dependent upon the upstream resistance.

MSFP by itself does not determine flow. Flow depends upon the difference between MSFP and right atrial pressures, and the right atrial pressure is regulated by the heart and - another key concept in Guyton's analysis by the venous resistance. The pressure drop from the compliant veins to the right heart is small but crucial because this pressure drop is one of the major determinants of the emptying of venous blood back to 


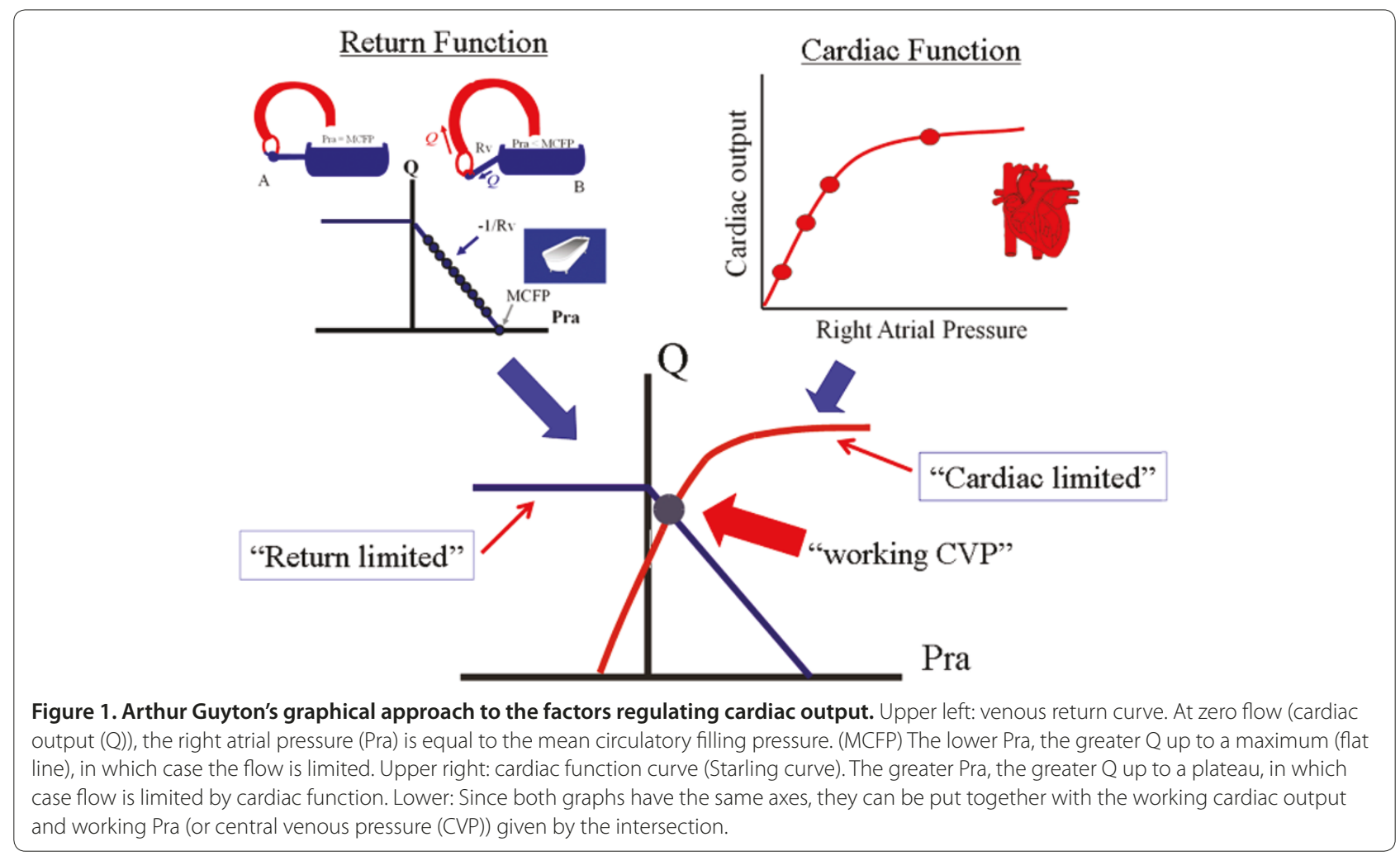

the heart. Although some have disputed the significance of venous resistance $[14,15]$ as a determinant of venous return and cardiac output, we used a computational model of the circulation to show that venous resistance can indeed have very important effects and including it can result in very different conclusions on outcome [16]. Our results were also consistent with Guyton's observations from a study in dogs in which he produced the equivalent of a marked decrease in left ventricular function [17]. These studies indicate the importance of considering the effects of drugs on venous resistance. Nitroglycerine and nitroprusside $[18,19]$ as well as $\beta$ adrenergic agonists can decrease venous resistance [20], and these drugs will increase venous return with a constant stressed volume. Venous resistance can be increased by $\alpha$-adrenergic agonists such as phenylephrine, which will decrease cardiac output. This explains why phenylephrine can raise arterial pressure but does not usually increase cardiac output [13].

In summary, Arthur Guyton identified four determinants of venous return: stressed volume, venous compliance, venous resistance, and right atrial pressure. He graphically represented the return function by putting right atrial pressure on the $x$ axis, because right atrial pressure is regulated by the function of the heart, and he put venous flow, which in the steady state is equal to the cardiac output, on the $y$ axis (Figure 1). When venous flow is zero, the right atrial pressure equals MSFP and, depending upon how flow is stopped and how much time is allowed for equilibrium of all compartments, it also can equal MCFP. Lowering the right atrial pressure allows venous blood to return to the heart. The lower the right atrial pressure, the greater the venous return up to a maximum flow. This limit to venous return occurs when the pressure inside vessels entering the thorax is less than the pressure outside, which limits but does not stop flow because the vessels flutter between opening and closing in what is called a vascular waterfall [21].

The axes of Guyton's plot of venous return are the same as the same axes of Starling's cardiac function curve. Cardiac and venous return functions can thus be plotted on the same graph and their intersection gives the solution for how these two functions determine cardiac output (Figure 1). This plot identifies two limits to the circulation. There is a plateau to the cardiac function relationship. When the venous return curve intersects this region of the cardiac function curve, giving more volume will not increase cardiac output because the volume simply shifts the venous curve to the right, which increases right atrial pressure but does not appreciably increase end-diastolic volume. On the left side of the relationship, when the heart is functioning on the flat part of the venous return curve, increasing cardiac function - including increasing the heart rate - does not 
increase cardiac output. Under this condition cardiac output can only be increased by increasing the stressed volume or decreasing venous resistance. This also brings up a key implication of Guyton's work. The heart can never pump out more than it receives from the venous reservoir, and the volume the heart receives is limited by venous collapse. When collapse occurs it becomes evident that circuit factors determine the maximum possible cardiac output because any value of right atrial pressure above the venous collapse pressure actually decreases the return of blood to the heart.

Although it is not possible at the bedside to obtain fully developed cardiac function-venous return curves, the general concepts can still be very helpful for understanding the physiological significance of changes in cardiac output that occur with changes in right atrial pressure (or central venous pressure (CVP), which is essentially synonymous with right atrial pressure), as will be discussed next.

Guyton's graphical analysis of the interaction of venous return and cardiac function makes it clear that a single value of CVP cannot predict blood volume or cardiac status. For example, low CVP is the norm in healthy individuals. In the resting upright posture, CVP usually is less than atmospheric pressure because of the negative pressure in the thorax at functional residual capacity and optimal cardiac function (Figure 2). On the contrary, a low CVP can be seem in someone with loss of volume and low cardiac output and normal or even impaired cardiac function.

\section{A Guyton-guided approach to the management of shock and hemodynamic monitoring}

A good place to begin is by considering the relationship of pressure to flow as described by Poieseuille's law, which indicates that blood pressure is approximately equal to the product of cardiac output and systemic vascular resistance (Figure 3). This simple relationship indicates that a low blood pressure must be explained by a decrease in cardiac output or a decrease in systemic vascular resistance. Since vascular resistance is calculated, the variable one needs to know is cardiac output. If blood pressure is low, and cardiac output is normal or elevated, the primary reason for low blood pressure is that the systemic vascular resistance is low. If the cardiac output is decreased this can - based on Guyton's graphical analysis of the circulation - be due to a decrease in cardiac function or a decrease in the venous return function (Figure 4). Since the cardiac and venous return functions intersect at the right atrial pressure, the right atrial pressure/CVP helps to determine whether a decrease in cardiac function or a decrease in return function is the primary problem. If the CVP is high, the problem is primarily decreased cardiac function - and

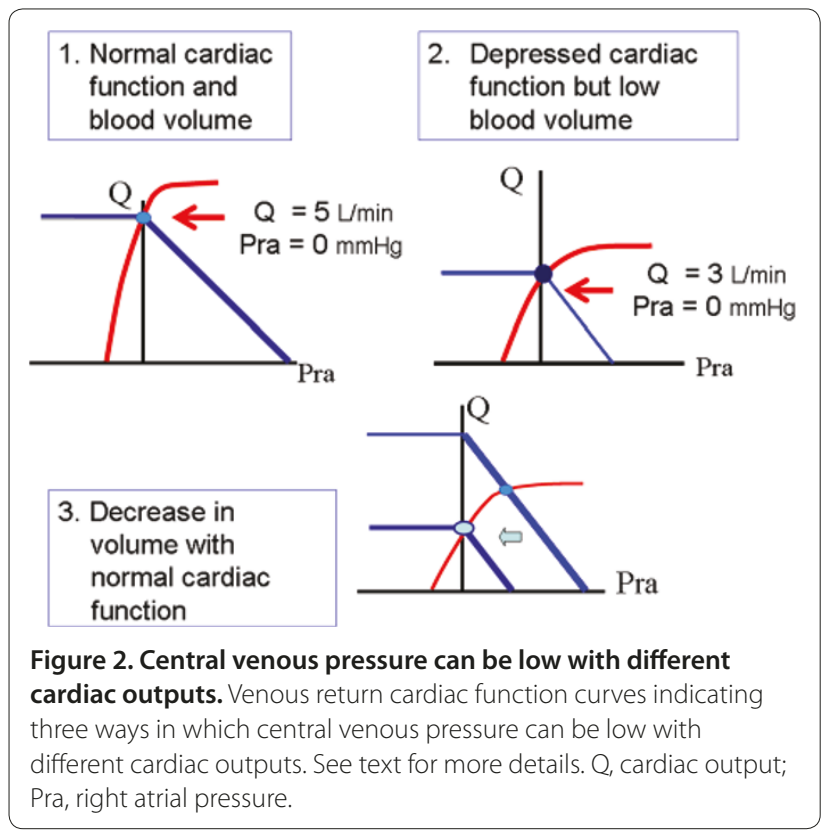

diagnostic and therapeutic interventions should be aimed at explaining why cardiac function is reduced. If the CVP is low (Figure 2), the primary problem is the venous return - and providing more volume will probably solve the problem.

The question then arising is what constitutes a high CVP. The plateau of the cardiac function curve can occur even at low values of CVP [22] (Figure 2) but, as a useful guide, when CVP $>10 \mathrm{mmHg}$ (that is, when the reference level is $5 \mathrm{cmH}_{2} \mathrm{O}$ below the sternal angle, or $\sim 13 \mathrm{mmHg}$ relative to the mid-axillary line) the probability of an increase in cardiac output in response to a fluid bolus is low, unless there is high positive end-expiratory pressure, longstanding pulmonary hypertension, or high intraabdominal pressure [22]. When one is not sure whether volume might help, the solution is to give a fluid challenge. Essential components of this challenge are that sufficient fluid is given to raise the CVP by $2 \mathrm{mmHg}$ or more so that it is clear Starling's law has been tested and then whether or not there is an increase in cardiac output is monitored. This is most easily done when cardiac output is directly measured, in which case an increase in cardiac index in the range of $0.3 \mathrm{l} / \mathrm{minute} / \mathrm{m}^{2}$ can be considered to indicate that the heart is volume responsive and that the change is not just measurement variation. If the triggers for giving volume are still not corrected, boluses can be repeated until the triggers are corrected or until there is no further increase in flow with the volume despite an increase in CVP. If the CVP rises by $2 \mathrm{mmHg}$ and the cardiac index does not change, however, it is very unlikely that further volume infusions will help and some other therapy is indicated. We successfully 


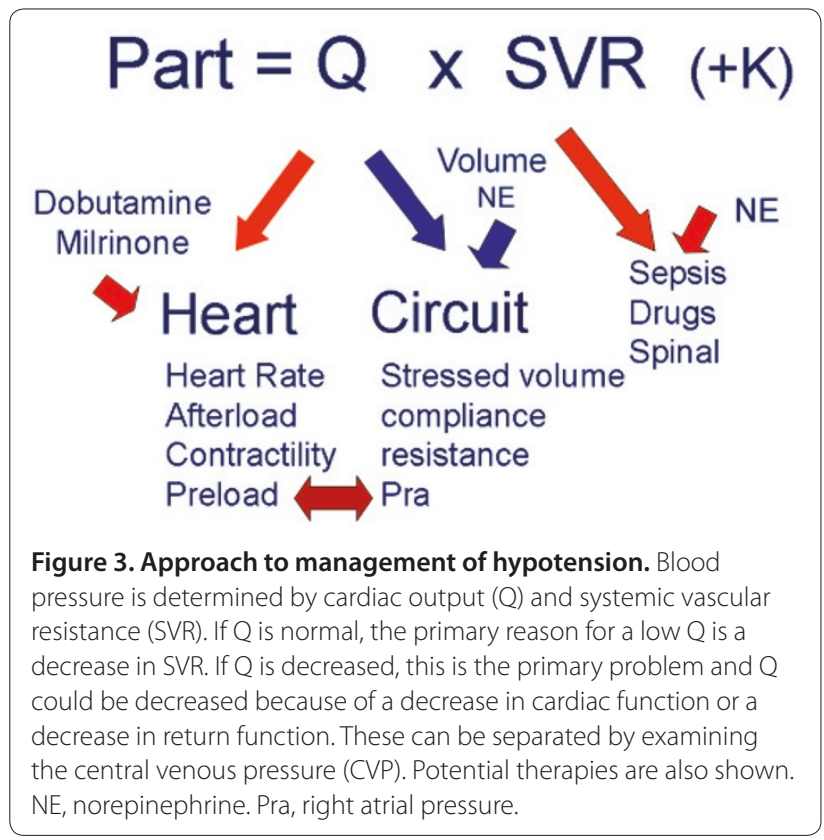

used an algorithm based on these principles to show that a colloid solution for resuscitation following cardiac surgery significantly reduced the need for catecholamines the morning after surgery compared with a crystalloid solution and with less fluid being given [23].

Measurements of CVP and cardiac output are most useful when there has been a change in a patient's clinical status (Figure 3). Based on Guyton's concepts, the direction of changes in cardiac output and CVP can be used to indicate the dominant process that has occurred. I say dominant because more than one process can happen at the same time. For example, there can be a decrease in cardiac function at the same time as there is a decrease in venous return. The analysis again starts with what happed to cardiac output. If blood pressure fell with a rise or no change in cardiac output, the primary problem is a decrease in systemic vascular resistance and, although increasing cardiac output may help to some extent, it may be necessary to pharmacologically increase systemic vascular resistance with a drug such as norepinephrine. If blood pressure fell with a fall in cardiac output, then the primary problem is the fall in cardiac output and the next question is whether cardiac output fell because of a decrease in cardiac function or in the return function. This is answered by examining the CVP. If cardiac output fell and CVP fell too, the primary problem is a decrease in the return function, which usually means that volume is needed. If the cardiac output fell with a rise in CVP, the primary problem was probably a decrease in cardiac function and use of inotropic drugs such as dobutamine, epinephrine or milrinone is indicated.

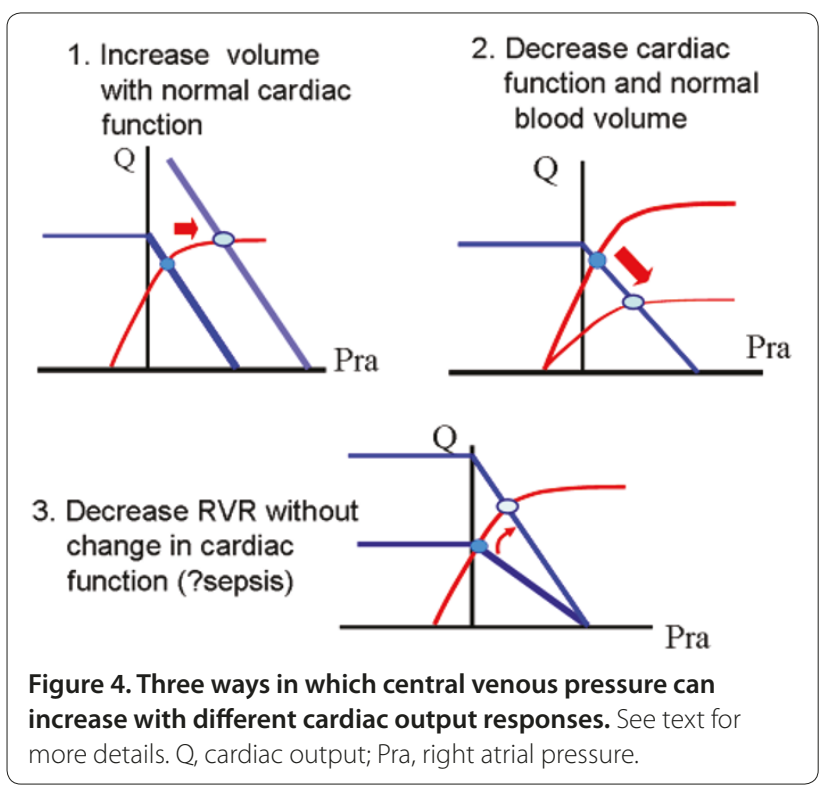

The approach outlined above requires measurement of cardiac output, or at least a surrogate, which limits its utility. At the more basic level, clinical signs of adequate cardiac output can often be sufficient and high CVP values can even be identified in many patients by examining jugular venous distension or inferior vena cava diameter by echocardiography. If the patient has a clear sensorium, warm extremities, and normal renal function, cardiac output probably is adequate for tissue needs. The next level of assessment involves obtaining laboratory values that support adequate tissue perfusion and cardiac output. These include blood lactate, normal base excess - or even a negative base excess if it can be explained by elevated chloride - or central venous oxygen saturation. When normal, these values support adequate tissue perfusion but do not rule out inadequate tissue perfusion, especially a normal central venous saturation; however, abnormal numbers almost always indicate insufficient cardiac output for tissue needs. Until recently, measurement of cardiac output required the use of a pulmonary artery catheter, but there are now a number of less invasive and even completely non-invasive devices that can be used to at least analyze the dynamic response to a fluid challenge or inotropic or vasoactive drug [24,25]. This analysis should allow algorithms in the future that have a physiological approach to hemodynamic management and are based on Guyton's analysis of the circulation.

\section{Inspiratory variations in central venous pressure}

An example of 'applied Guyton' at the bedside comes from the use of respiratory variations in CVP to predict fluid responsiveness (Figure 5) [26]. During spontaneous 


\section{Inspiratory fall in Pra Inspiratory fall in Pra}
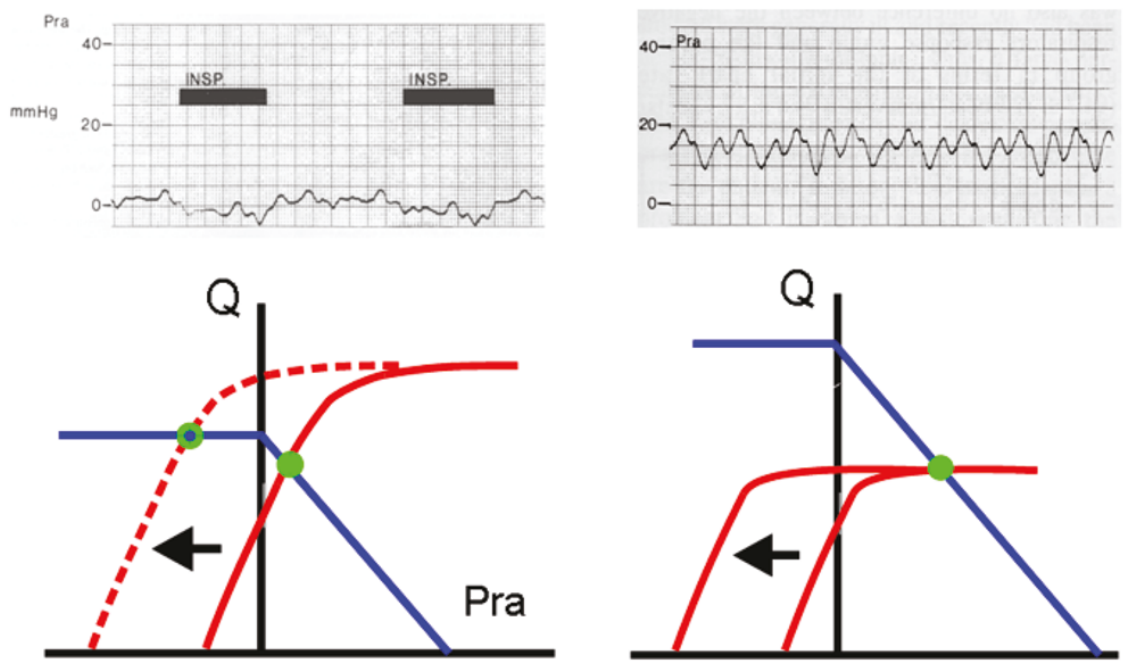

Figure 5. Inspiratory variations in central venous pressure predict response of cardiac output to a volume infusion. Patients who have an inspiratory (insp.) fall in central venous pressure (CVP) (left side) can have an increase in cardiac output (Q) with a volume infusion, but not always because it depends how close to the plateau of the cardiac function the venous return intersects. Patients who do not have an inspiratory fall in CVP are unlikely to respond to a volume infusion because this indicates that the venous return curve is intersecting the flat part of the cardiac function curve. Pra, right atrial pressure.

inspiratory efforts, whether the person is breathing unassisted or is intubated and mechanically ventilated, the pleural pressure falls. Importantly, the hydrostatic pressures measured with bedside transducers are made relative to a reference level and a reference pressure value. Since the transducers are surrounded by atmospheric pressure, hemodynamic pressures are referenced relative to atmospheric pressure and a level that is relative to the mid-point of the right heart [27]. However, the heart is not surrounded by atmospheric pressure, but rather by pleural pressure, and the pressure that counts for the preload of the heart is the pressure inside the heart relative to the outside.

To use Guyton's graphical approach during the respiratory cycle, the cardiac function curve must therefore move relative to the venous return curve to account for the changing surrounding pressure. During a negative pleural pressure inspiration this means that the cardiac function curve moves to the left of the venous return curve. If the venous return curve intersects the ascending part of the cardiac function curve, CVP falls during inspiration relative to atmospheric pressure (Figure 5) and output from the right heart transiently increases because the heart is effectively lowered relative to the venous reservoir, which allows more blood to come back to the heart. However, if the venous return curve intersects the plateau portion of the cardiac function curve, CVP does not change during inspiration (Figure 5). The absence of an inspiratory fall in CVP when there is a fall in pleural pressure thus indicates that the patient's heart is functioning on the plateau of the cardiac function curve and should not respond to fluids. In a prospective trial we found that this was indeed the case [26].

There are some caveats to this approach. Pleural pressure must fall sufficiently to know whether or not the patient is on the flat part of the curve. If conscious, the person can be asked to make a stronger effort to test this situation. Second, the test is most useful in the negative sense. That is, lack of an inspiratory fall in CVP indicates that the patient is unlikely to respond, whereas the presence of a fall does not indicate that the patient will respond because it depends on how close the intersection of venous return curve is to the plateau of the cardiac function curve. Finally, it is important to rule out whether the fall in CVP is actually due to the release of an active expiration and use of abdominal muscles. This is recognized by noting whether or not there is a rise in CVP during expiration.

\section{Effect of positive pressure}

An implication of Guyton's cardiac function-venous return analysis is that the cardiac function curve is shifted to the right with positive-pressure breathing. If the venous return curve intersects the ascending part of the cardiac function curve, cardiac output will fall and the CVP will rise with the positive pleural pressure. However, if the venous return curve intersects the flat part of the cardiac function curve, there could be an 
increase in pleural pressure without a fall in cardiac output. This would indicate that patients whose CVP falls with a negative-pressure breath should always have a fall in cardiac output when positive pleural pressure is applied because they should be operating on the ascending part of the cardiac function curve. On the other hand, patients who do not have an inspiratory fall in CVP may or may not have a fall in cardiac output depending on how much the cardiac function curve is shifted to the right of the venous return curve with the increase in pleural pressure.

We tested these predictions in patients who had spontaneous inspiratory efforts and in whom positive endexpiratory pressure was going to be increased [28]. The test worked for the groups but had a low predictive value in individual patients, most probably because the positive pleural pressure triggered reflex adjustments that changed the volume status by recruiting unstressed volume into stressed volume. Furthermore, the magnitude of these adjustments depends upon the reserves in vascular capacitance $[29,30]$. In contrast, the variations during single breaths that we used for volume responsiveness are too short for there to be reflex adjustments.

\section{Conclusion}

Guyton provided a comprehensive analysis of the interaction of cardiac function and the venous return function. He showed that the cardiac output and CVP values are determined by the intersection of these two functions. Knowledge of these processes can help guide clinicians in their resuscitative strategies for treatment of hemodynamic instability. Guyton's concepts help one understand the limits of the cardiovascular system and thus what is physiologically possible. The development of newer devices for measuring cardiac output will make direct application of these principles more feasible.

\section{Abbreviations}

CVP, central venous pressure; MCFP, mean circulatory filling pressure; MSFP mean systemic filling pressure.

\section{Competing interests}

The author declares that he has no competing interests.

Published: 29 October 2012

\section{References}

1. Rushmer RF, Smith OA, Jr: Cardiac control. Physiol Rev 1959, 39:41-68.

2. Guyton AC: Determination of cardiac output by equating venous return curves with cardiac response curves. Physiol Rev 1955, 35:123-129.

3. Guyton AC, Lindsey AW, Bernathy B, Richardson T: Venous return at various right atrial pressures and the normal venous return curve. Am J Physiol 1957, 189:609-615.

4. Guyton AC, Polizo D, Armstrong GG: Mean circulatory filling pressure measured immediately after cessation of heart pumping. Am J Physiol 1954, 179:261-267

5. Coleman TG, Manning RD, Jr, Norman RA, Jr, Guyton AC: Control of cardiac output by regional blood flow distribution. Ann Biomed Eng 1974, 2:149-163.
6. Magder S: The classical Guyton view that mean systemic pressure, right atrial pressure, and venous resistance govern venous return is/is not correct. J Appl Physiol 2006, 101:1523-1525.

7. Magder S, De Varennes B: Clinical death and the measurement of stressed vascular volume. Crit Care Med 1998, 26:1061-1104.

8. Rothe CF: Reflex control of veins and vascular capacitance. Physiol Rev 1983, 63:1281-1295.

9. Deschamps A, Magder S: Baroreflex control of regional capacitance and blood flow distribution with or without alpha adrenergic blockade. J App/ Physiol 1992, 263:H1755-H1763.

10. Magder S, Veerassamy S, Bates $\mathrm{JH}$ : A further analysis of why pulmonary venous pressure rises after the onset of LV dysfunction. J Appl Physio/ 2009, 106:81-90.

11. Thiele RH, Nemergut EC, Lynch C III: The physiologic implications of isolated $a_{1}$ adrenergic stimulation. Anesth Anal 2011, 113:284-296.

12. Thiele RH, Nemergut EC, Lynch C III: The clinical implications of isolated $a_{1}$ adrenergic stimulation. Anesth Analg 2011, 113:297-304.

13. Magder S: Phenylephrine and tangible bias. Anesth Analg 2011, 113:211-213.

14. Brengelmann GL: Counterpoint: the classical Guyton view that mean systemic pressure, right atrial pressure, and venous resistance govern venous return is not correct. J App/ Physio/ 2006, 101:1525-1526.

15. Brengelmann $G L$ : A critical analysis of the view that right atrial pressure determines venous return. J Appl Physio/ 2003, 94:849-859.

16. Burkhoff $D$, Tyberg JV: Why does pulmonary venous pressure rise after onset of left ventricular dysfunction: a theoretical analysis. Am J Physiol 1993, 265:H1819-H1828.

17. Lindsey AW, Guyton AC: Continuous recording of pulmonary blood volume: pulmonary pressure and volume changes. Am J Physiol 1959, 197:959-962.

18. Pouleur H, Covell JW, Ross J, Jr: Effects of nitroprusside on venous return and central blood volume in the absence and presence of acute heart failure. Circulation 1980, 61:328-337.

19. Ogilvie Rl: Effect of nitroglycerin on peripheral blood flow distribution and venous return. J Pharmacol Exp Ther 1978, 207:372-380.

20. Green JF: Mechanism of action of isoproterenol on venous return. Am J Physiol 1977, 232:H152-H156.

21. Permutt S, Riley S: Hemodynamics of collapsible vessels with tone: the vascular waterfall. J Appl Physiol 1963, 18:924-932.

22. Magder S, Bafaqeeh F: The clinical role of central venous pressure measurements. J Intensive Care Med 2007, 22:44-51.

23. Magder S, Potter BJ, Varennes BD, Doucette S, Fergusson D: Fluids after cardiac surgery: a pilot study of the use of colloids versus crystalloids. Crit Care Med 2010, 38:2117-2124

24. de Waal EE, Wappler F, Buhre WF: Cardiac output monitoring. Curr Opin Anaesthesiol 2009, 22:71-77.

25. Vincent JL, Rhodes A, Perel A, Martin GS, Della Rocca G, Vallet B, Pinsky MR, Hofer CK, Teboul JL, de Boode WP, Scolletta S, Vieillard-Baron A, De Backer D, Walley KR, Maggiorini M, Singer M: Clinical review: Update on hemodynamic monitoring - a consensus of 16. Crit Care 2011, 15:229.

26. Magder SA, Georgiadis G, Cheong T: Respiratory variations in right atrial pressure predict response to fluid challenge. J Crit Care 1992, 7:76-85.

27. Magder S: Central venous pressure: a useful but not so simple measurement. Crit Care Med 2006, 34:2224-2227.

28. Magder S, Lagonidis D, Erice F: The use of respiratory variations in right atrial pressure to predict the cardiac output response to PEEP. J Crit Care 2002, 16:108-114.

29. Nanas S, Magder S: Adaptations of the peripheral circulation to PEEP. Am Rev Respir Dis 1992, 146:688-693.

30. Fessler HE, Brower RG, Wise RA, Permutt S: Effects of positive end-expiratory pressure on the gradient for venous return. Am Rev Respir Dis 1992, 146:4-10.

doi:10.1186/cc11395

Cite this article as: Magder S: Bench-to-bedside review: An approach to hemodynamic monitoring - Guyton at the bedside. Critical Care 2012, 16:236. 